of the slip obtained on adding a small quantity of one of these substances. This permits a considerable amount of colloidal matter to be added to the solution with the substance, without a change in the fluidity, the amount of matter added affording a sort of measure of the increase in fluidity introduced by the dissolved substance. The latter effect is known and is used to obtain a concentrated porcelain slip for casting in the wet porcelain process by the addition of a small quantity of sodium silicate. It was found that the increase in compactness of the deposit on adding a small quantity of the foregoing substances was attended by an increase in the fluidity of the solution.

These two effects and their association may be explained by supposing that the dissolved substance decreases the "radii of the spheres of action" of the colloidal particles. The particles would accordingly, on addition of the substance, have additional room for motion with a resultant increase in the fluidity in the solution, and would pack more closely on being deposited by an electric current. A theoretical investigation along these lines is given.

It is evident that if the radii of the spheres of action of the particles are decreased sufficiently spontaneous precipitation would result. Thus it would follow from the foregoing results that a substance which precipitates a colloidal solution, if added in an appropriate amount, should in all cases increase the fluidity of the solution and increase the compactness of the deposit obtained with an electric current.

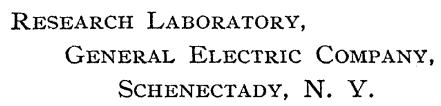

\title{
The Effective Range of $\beta$-Rays.
}

By J. A. Gray and A. V. Douglas.

WHEN plates of any substance are placed over a source of $\beta$-rays, a thickness of the substance will eventually be reached such that none of the original $\beta$-rays will pass through it. The smallest mass per unit area of the substance which will satisfy this condition is called the effective range of the $\beta$-rays in that substance. It is called effective range owing to the fact that, through scattering, $\beta$-rays really have a greater total path than that indicated by these measurements. Mass per unit area is taken instead of thickness so as to compare different substances.

This range has been obtained by using an electroscope to give a measure of the intensity of the $\beta$-rays. The source of $\beta$-rays was an active preparation of radium $(\mathrm{D}+\mathrm{E})$. The effective range of these $\beta$-rays has been found in paper, aluminium, copper, tin and lead. In paper the range is 0.47 gramme, in lead 0.34 gramme, the range being inversely proportional to what has been called the mass absorption coefficient of the $\beta$-rays.

The range in paper has also been obtained of rays scattered through large angles by sheets of silver and lead. Although the rays examined are slower, on the whole, than the primary rays, there is a strong indication that $\beta$-rays 
lose only a small proportion of their energy, when they are scattered through large angles. It is difficult to be quite certain that they lose any at all, because the scattering of $\beta$-rays is not a surface effect.

The effective ranges of secondary $\beta$-rays excited in lead by different types of $\mathrm{x}$ - and $\gamma$-rays have also been determined and are being found useful in problems connected with the transformation of $\beta$-rays into $\mathrm{x}$-rays and vice versa.

McGill University.

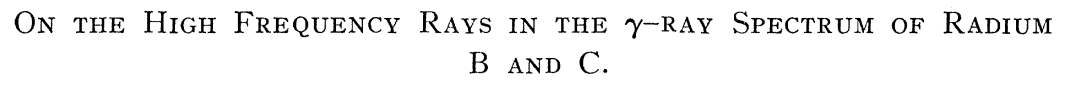

By Alois F. Kovarik.

THE method described was devised for the study of the spectra of soft $\gamma$-rays, for example, the $\gamma$-rays from radium DE-an investigation which is planned by the writer. However, since the penetrating radiations offer a better chance to test the method and also because the only work on record on the $\gamma$-ray spectrum of radium is by the photographic method ${ }^{1}$ it was thought advisable to study at first the spectrum of the $\gamma$-rays from radium B and C. The work here recorded is preliminary to a study of the whole spectrum and bears on the high frequency radiations alone.

The source of the radiations was a standard radium salt of $\mathbf{I} .3$ milligrams radium content. The radium was placed in the center of a lead block $15 \mathrm{~cm}$. on the edge and the rays passed through an opening in this block and through a slit $\mathrm{I} \mathrm{mm}$. wide and $6 \mathrm{~cm}$. deep in another block of lead placed in front of the former. The rays fell on a crystal of calcite and were reflected into a point discharge counting chamber after passing through a slit $0.5 \mathrm{~mm}$. wide and $7 \mathrm{~cm}$. deep placed in front of the counting chamber. The counting chamber was also surrounded by lead. The crystal and the counting chamber were moved together, the latter through double the angle of the former and the discharges produced in the counting chamber by the $\gamma$-rays entering through the slit (and otherwise) were automatically registered using the device previously described by the writer. ${ }^{2}$

The principal lines recorded by Rutherford were verified but other lines were also detected. Among these, it is of especial interest to note that there are at least five and possibly more lines of shorter wave-length than the shortest one (43' from rocksalt) reported by Rutherford and Andrade. The glancing angles from calcite of these lines are $4 \mathrm{I}^{\prime}$ (corresponding to the $43^{\prime}$ from rocksalt), $37 \cdot 5^{\prime}, 33^{\prime}, 27 \cdot 5^{\prime}, 21^{\prime}$, and $16^{\prime}$.

The study will be carried on, using crystals of various kinds and also of various thicknesses. The method is applicable in particular to the study of rays from weak sources, as is seen from the present case in which only a little more than a milligram of radium was used.

YALE UNIVERSITY.

${ }^{1}$ Rutherford and Andrade, Phil. Mag., 27, 854; 28, 263, I9r4.

2 Kovarik, Phys. Rev., (2), xviii., 272, I9I9. 